\title{
Experiencia sobre el uso del Kahoot como herramienta dinamizadora y de mejora en la asignatura de Gestión Empresarial en Ingeniería
}

\author{
Bouza Fernández, María Sonia \\ Universidade da Coruña, Departamento de Ingeniería Naval e Industrial, Escuela \\ Politécnica Superior, 0000-0003-3325-6363
}

\section{RESUMEN}

Este trabajo detalla la experiencia del uso de la herramienta de gamificación Kahoot en la asignatura de Gestión Empresarial en las titulaciones de Ingeniería de la Escuela Universitaria Politécnica. Esta asignatura se trata de una materia que se imparte en el $1^{0}$ cuatrimestre de $1^{0}$ curso y que presenta ciertas dificultades para su impartición, al tratarse de una materia que gran parte del alumnado no ha cursado hasta ese momento y no le encuentran relación con la titulación que estudian, lo que genera desmotivación. Los objetivos de este estudio eran, por un lado, analizar los efectos que ha tenido la técnica de gamificación empleada en el proceso de aprendizaje de los estudiantes y por otro conseguir la dinamización de las clases magistrales. Los resultados obtenidos han sido satisfactorios tanto para los alumnos como para el docente, dado que permitió mejorar los resultados académicos y conseguir que los alumnos fueran más constantes en el seguimiento de la asignatura.

PALABRAS CLAVE: Gamificación, Kahoot, docencia universitaria, ingeniería. 


\section{CITA RECOMENDADA:}

Bouza Fernández, María Sonia (2020): Experiencia sobre el uso del Kahoot como herramienta dinamizadora y de mejora en la asignatura de Gestión Empresarial en Ingeniería. En De la Torre Fernández, E. (ed.) (2020). Contextos universitarios transformadores: Boas prácticas no marco dos GID. IV Xornadas de Innovación Docente. Cufie. Universidade da Coruña. A Coruña (págs. 33-42). DOI capítulo: https://doi.org/10.17979/spudc.9788497497756.033

DOI libro: https://doi.org/10.17979/spudc.9788497497756

\section{ABSTRACT}

This work details the experience of using the Kahoot gamification tool in the subject of Business Management in the Engineering degrees of the Polytechnic University School. This subject is a subject that is taught in the 1st semester of the 1st year and that presents certain difficulties for its teaching, since it is a subject that a large part of the student body has not taken until that moment and they do not find it related to the degree that study, which generates demotivation. The objectives of this study was, on the one hand to analyze the effects that the gamification technique used in the student learning process has had and on the other to achieve the dynamization of the master classes. The results obtained have been satisfactory both for the students and for the teacher, since it allowed to improve the academic results and make the students more constant in the monitoring of the subject.

KEY WORDS: Gamification, Kahoot, university teaching, engineering. 


\section{INTRODUCCIÓN}

En la sociedad en la que vivimos, y sobre todo en la generación de los actuales estudiantes universitarios, conocidos como nativos digitales, el uso del teléfono móvil se ha generalizado, siendo utilizado en casi todos los ámbitos de la vida. Es por ello, que la educación debe adaptarse al uso de estos dispositivos en el aula, como otra herramienta más, en vez de verlo como algo que hay que prohibir o erradicar.

Los smartphones de hoy en día prestan casi las mismas posibilidades que una tablet, lo que ha permitido que algunos softwares hayan desarrollado una versión para el teléfono móvil y dispongamos de una gran variedad de apps.

El término gamificación es un término que ha surgido en la última década, habiéndose generalizado su uso a partir del año 2010.

Zichermann en 2011 define la gamificación como "el proceso por el cual se aplican los planteamientos y las mecánicas de los juegos para involucrar a los usuarios".

Algunos autores como Sailer, Hense, Mandl \& Kleevers (2017) consideran la motivación como el elemento más influyente en la gamificación para la enseñanza.

King, Song, Lockee \& Bueton (2018) define la gamificación como "un conjunto de actividades y procesos para resolver problemas al usar 0 aplicar las características de los elementos del juego"

Existen diferentes definiciones, en función del enfoque, pero todas confluyen en considerar la motivación como un pilar para el aprendizaje.

La motivación, actúa como factor para que los alumnos perciban la asignatura de otra forma, al utilizar técnicas en el aula que permitan dinamizar las clases y las hagan más atractivas y les faciliten el estudio. Se trata de cambiar la actitud del alumno hacia esa asignatura sobre la que que desde un principio tienen un rechazo.

Por otro lado, cabe diferenciar entre la gamificación y los juegos educativos, tal y como establece Kapp (2012), la primera muestra un espacio de juego mucho más atractivo y motivante para los jugadores que la segunda. 
Existen múltiples herramientas de gamificación, de las cuáles cabe destacar:

- Kahoot: Herramienta de origen noruego que surgió en el año 2011. Se basa en el empleo de cuestionarios o discusiones (denominados kahoots), obteniendo un feedback al instante. Está formada por dos plataformas, una para la creación de los cuestionarios y otra para jugar. Como ventajas que plantea es que los jugadores no requieren registrarse en la aplicación, el docente puede usar los kahoots elaborados por otros docentes en todo el mundo y se puede utilizar en cualquier dispositivo (tablet, móvil o portatil).

- Socrative: aplicación que surge en el año 2010 en EEUU y que se emplea para realizar cuestionarios en tiempo real. Es similar al Kahoot.

- Plickers: en esta herramienta los estudiantes responden levantando la tarjeta que contiene el código en la posición correcta y el docente escanea con la aplicación las mismas.

- Quizizz: Permite crear cuestionarios que los alumnos pueden responder en un juego directo, como tarea o de manera individual. Permite seleccionar que el tiempo de respuesta no valga puntos a diferencia de las dos primeras herramientas. Permite incorporar imágenes no sólo en las preguntas sino también en las respuestas.

El objetivo fundamental de estas herramientas es motivar al alumno para conseguir tener una mayor participación en el aula y un mayor seguimiento de la asignatura y que por lo tanto mejoren los resultados académicos.

Las empresas fueron pioneras en el uso de herramientas de gamificación, aplicándolas dentro de las diferentes áreas de la organización (marketing, recursos humanos...), experiencias muchas de ellas muy exitosas tal y como se recogen en Gallego \& Pablos, (2013) donde se describen prácticas de gamificación en el entorno empresarial y los resultados obtenidos. Pero el fenómeno de la gamificación no ha dejado indiferente a las prácticas formativas universitarias de distintas titulaciones, tal y como se recoge en Eguia (2017) y en Kim (2018). 


\section{CONTEXTUALIZACIÓN}

La experiencia que se presenta se lleva a cabo durante el curso 2018/2019 con estudiantes de Gestión Empresarial de la Escuela Universitaria Politécnica (EUP) de la Universidade da Coruña. Esta materia es de formación básica de 6 créditos ECTS que se imparte en el primer cuatrimestre del primer curso de las titulaciones del Grado en Ingeniería Eléctrica y del Grado en Ingeniería en Electrónica Industrial y Automática.

El temario de esta materia se divide en cuatro bloques, y cada bloque se compone de un número diferente de temas. La evaluación se realiza mediante prueba objetiva, trabajos tutelados y resolución de problemas. Se realizan dos exámenes parciales, uno para los bloques I y II y otro para el resto de bloques, que evalúan tanto la parte teórica como la parte práctica de esta materia. En concreto, la parte de teoría se evalúa con exámenes tipo test.

Como docente, me encuentro con la problemática de que los alumnos perciben esta materia como que no tiene relación alguna con la titulación en que se han matriculado. Si a esto le sumamos que la inmensa mayoría no la han cursado en sus estudios previos, a diferencia del resto de asignaturas de formación básica de primer curso (matemáticas, física, química.), provoca que el alumnado afronte la materia desde su comienzo con desmotivación.

Es por ello que con esta experiencia se fijaron como objetivos:

- Motivar al alumno.

- Dinamizar las clases magistrales.

- Analizar los resultados obtenidos con la técnica de gamificación empleada en el proceso de aprendizaje de los estudiantes.

utilizando como instrumento para este análisis la herramienta de gamificación Kahoot. Se optó por esta herramienta por ser muy intuitiva y más atractiva para el alumnado que la plataforma Moodle o los formularios de Microsoft Forms. 


\section{DESCRICIÓN DE LA EXPERIENCIA}

Se elaboraron cuestionarios Kahoots para cada uno de los bloques de la materia. Estos cuestionarios estaban compuestos entre 20-30 preguntas tipo test. Una vez en el aula, el alumnado hacía uso de sus móviles para dar respuesta a las cuestiones planteadas, identificándose con un nick que se correspondía con las iniciales de su nombre y apellidos.

A medida que los alumnos responden a las preguntas, se va obteniendo la retroalimentación del cuestionario en la pantalla del aula: número de alumnos que acertaron la pregunta, y número de los que la fallaron. Al mismo tiempo, la aplicación genera un ranking de posiciones en los que valora no sólo el acertar la pregunta sino también la velocidad en la respuesta. Esto generaba una rivalidad "sana" entre el alumnado. Seguidamente se reforzaban los conocimientos tratados en la pregunta.

Una vez finalizado el curso, se elaboró un cuestionario para analizar la percepción que tenían los alumnos sobre el uso de esta herramienta de gamificación.

Por otro lado, se decidió que estas actividades no formaran parte del sistema de evaluación de la asignatura, al tratarse de una prueba piloto.

\section{RESULTADOS}

Esta herramienta permitió en primer lugar, detectar, en función de los resultados de los kahoots, aquellos contenidos de la materia que no fueran asimilados por el alumnado o que resultaran difíciles de entender. Como consecuencia de esto, se revisó y mejoró el material docente, que se ponía a disposición de los estudiantes.

Se recabó información sobre la opinión del alumnado sobre esta herramienta utilizada a través de una encuesta breve que se realizó al finalizar la materia. La encuesta constaba de las siguientes preguntas:

- ¿Consideras que el Kahoot permite dinamizar las clases magistrales?

- ¿Estarías dispuesto a que Kahoot puntuase para la evaluación de la asignatura?

- ¿Consideras que el Kahoot es útil? 
- ¿Consideras que el Kahoot te ayudó a afianzar los contenidos de la materia?

Los resultados obtenidos en la encuesta se muestran en la figura 1. Estos datos reflejan que la inmensa mayoría de los alumnos consideran que la herramienta utilizada es útil y que permite dinamizar las clases magistrales, alcanzando así los dos primeros objetivos definidos en esta experiencia: la motivación del alumnado y la dinamización de las clases magistrales.

Pero en contraposición, no todos estarían dispuestos a que los resultados del Kahoot formaran parte del sistema de evaluación de la asignatura.

Consideras que el Kahoot permite dinamizar las clases magistrales

Estarías dispuesto a que el Kahot puntuase para la nota final

Consideras que el Kahoot es útil

Consideras que el Kahoot te ayudó a afianzar los conceptos de la materia

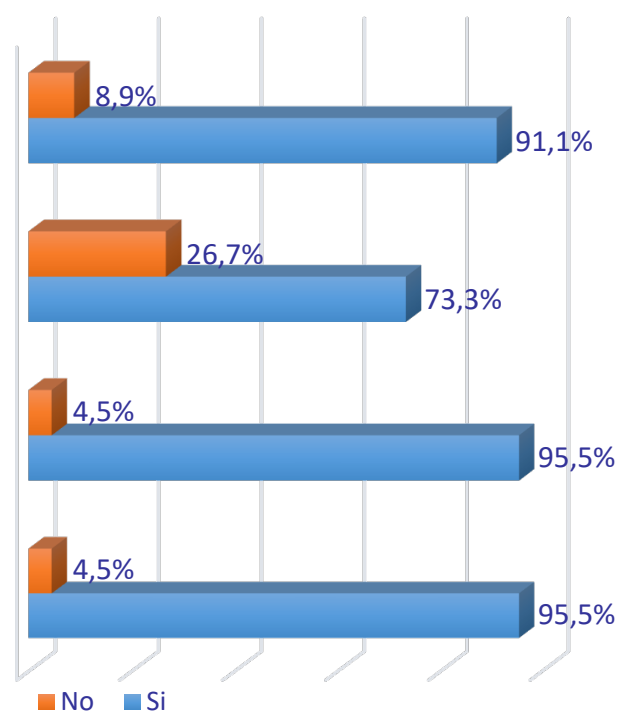

No $\quad \mathrm{Si}$

Figura 1. Repuestas del alumnado a la encuesta.

Para analizar los resultados en el proceso de aprendizaje, se compararon los datos obtenidos de las tasas de éxito, rendimiento y evaluación en ese curso con los resultados en el curso anterior. Estos resultados se muestran en las figuras 2 y 3 , separando los datos por titulaciones en que se imparte la materia. La valoración del proceso de aprendizaje es muy positiva, ya que se permitió reducir el número de abandonos de la materia y mejorar los resultados académicos. 


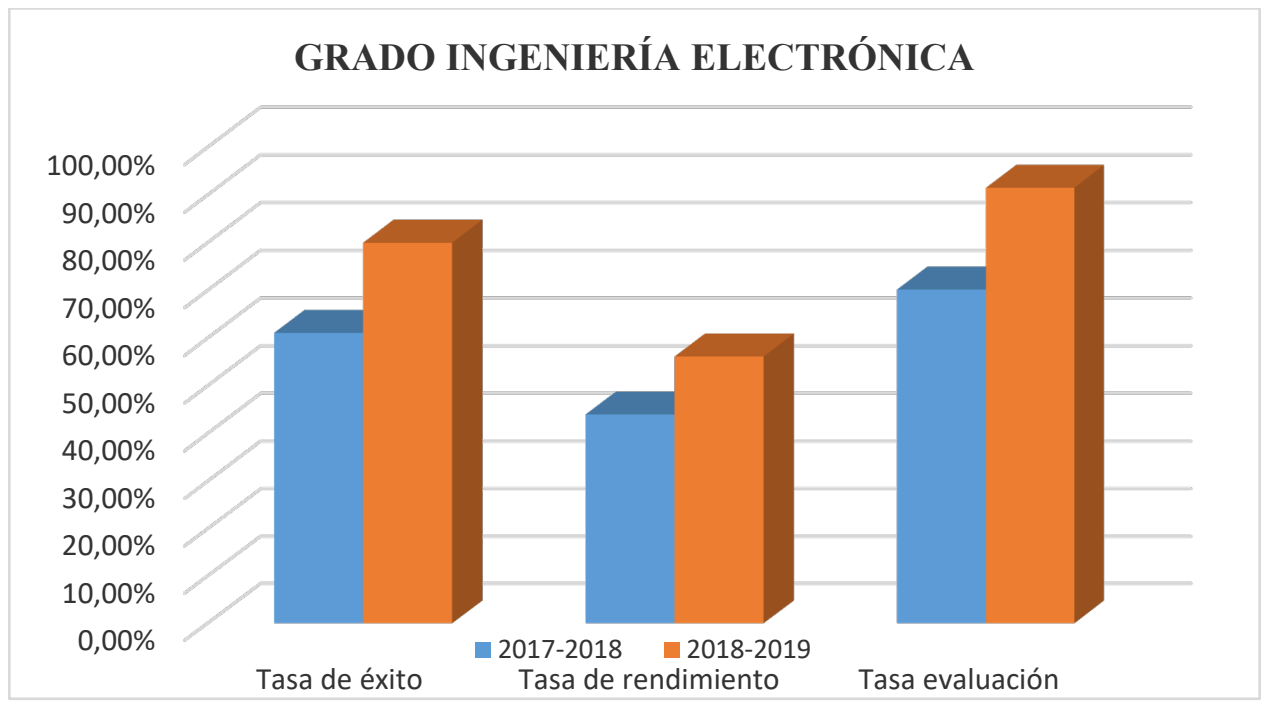

Figura 2. Tasas de éxito, tasa de rendimiento y tasa de evaluación de Gestión Empresarial en el Grado de Ingeniería Electrónica Indutrial y Automática

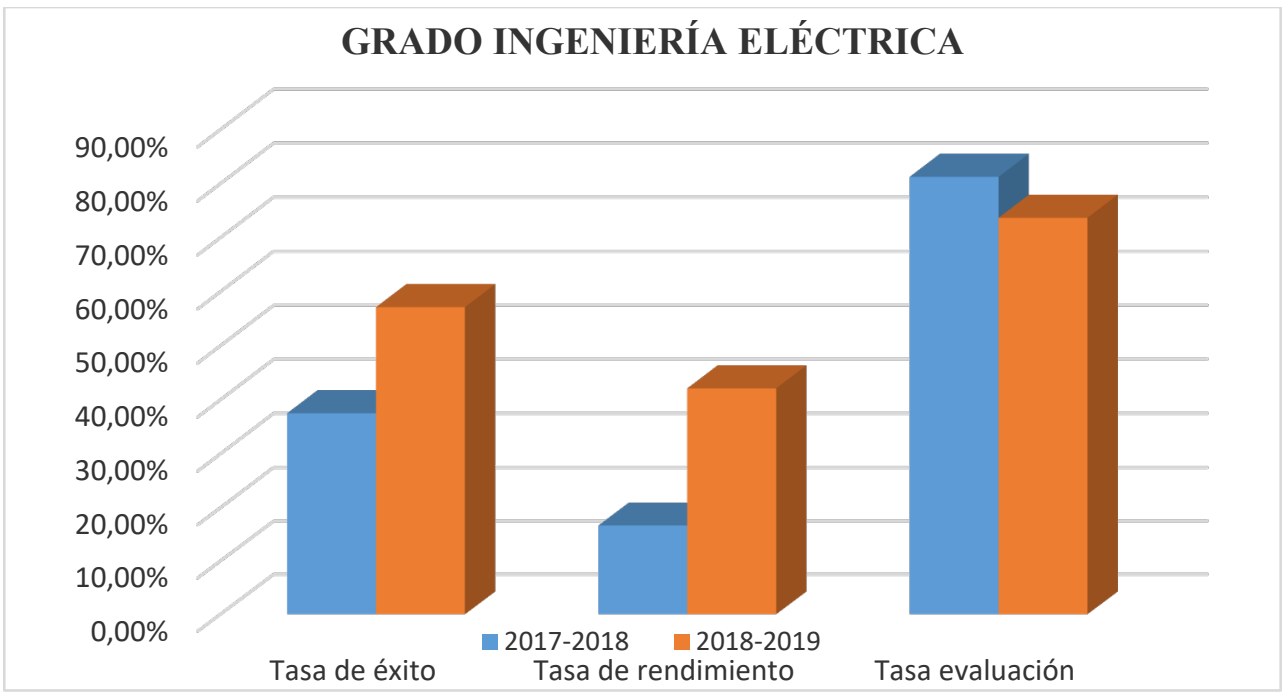

Figura 3. Tasas de éxito, tasa de rendimiento y tasa de evaluación de Gestión Empresarial en el Grado de Ingeniería Eléctrica. 


\section{CONCLUSIONES}

Es momento para que los docentes nos actualicemos y aprovechemos las ventajas que pueden aportar a la docencia el uso tanto de aplicaciones TIC como del uso de los dispositivos móviles en el aula.

Finalmente, este estudio permite concluir que el uso del Kahoot permitió cambiar la actitud de Ios estudiantes, al mostrar un mayor interés, participación y sobre todo un mayor seguimiento de la asignatura a medida que se avanzaba en la materia, lo que se reflejó en una mejora de los resultados. Por tanto, el incluir metodologías activas, haciendo uso de la tecnología, permite mejorar el proceso de enseñanza-aprendizaje.

\section{REFERENCIAS}

Eguía, J.L, Contreras Espinosa, R.S., Contreras Espinosa, R., Revuelta Domínguez, F.I, Guerra Antequera, J., Pedrera Rodríguez, M.I,... \& Paula, O.D.(2017). Experiencias de gamificación en aulas. Barcelona: Institut de la Comunicació, Universidad Autónoma de Barcelona.

Gallego Gómez, C., Pablos Heredero, C. (2013). "La gamificación y el enriquecimiento de las prácticas de innovación en la empresa: un análisis de excperiencias".Intangible Capital, 9 (3), pp. 800-822.

Kapp, K.M (2012). The Gamification of learning and instruction: Game-based Methods and Strategies for Training and Education. International Journal of Gaming and ComputerMediated Simulations, 4(4), pp.3.

Kim, S.; Song, K., Lockee, B. \& Burton, J. (2018). What is gamification in learning an education?. Gamification in Learning and Education, Springer International Publishing, pp. 25-38.

Kim, S.; Song, K., Lockee, B. \& Burton, J. (2018). Gamification Cases in Education. Gamification in Learning and Education, Springer International Publishing, pp.117-123. 
Cortizo, J.C., Carrero, F.M., Monsalve, B., Velasco, A., Díaz, L.I. \& Pérez-Martín, J. (2011). Gamificación y docencia: Lo que la Universidad tiene que aprender de los Videojuegos. Madrid: Universidad Europea de Madrid. Accesible en http://hdl.handle.net/11268/1750.

Sailer,M., Hense,J., Mandl,H. \& Klevers, M. (2017). Fostering development of work competences and motivation via gamification. En M. Mulder (Ed.), Competence-based Vocational and Professional Education (pp.795-818). Switzerland: Springer International Publishing AG.

Zichermann, G. \& Cunningham, C. (2011). Gamification by design: Implementing game mechanics in web and mobile apps. 0 Reilly Media Inc. 